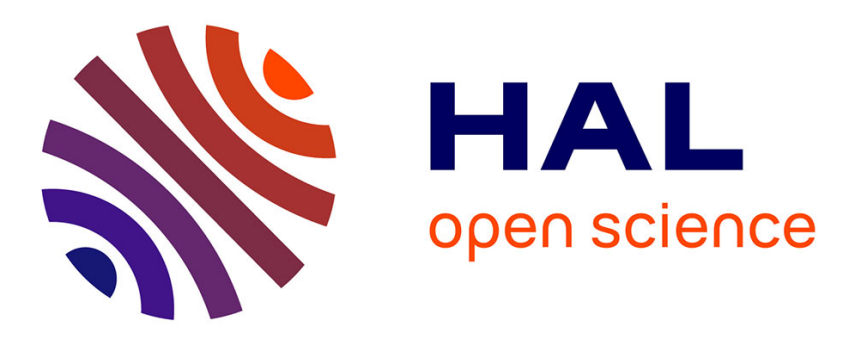

\title{
Une évaluation expérimentale des modes électroniques de résolution des litiges
}

Yannick Gabuthy, Nicolas Jacquemet

\section{To cite this version:}

Yannick Gabuthy, Nicolas Jacquemet. Une évaluation expérimentale des modes électroniques de résolution des litiges. Revue Economique, 2007, 58 (6), pp.1309-1330. 10.3917/reco.586.1309 . halshs00259457

\section{HAL Id: halshs-00259457 \\ https://shs.hal.science/halshs-00259457}

Submitted on 9 Nov 2009

HAL is a multi-disciplinary open access archive for the deposit and dissemination of scientific research documents, whether they are published or not. The documents may come from teaching and research institutions in France or abroad, or from public or private research centers.
L'archive ouverte pluridisciplinaire HAL, est destinée au dépôt et à la diffusion de documents scientifiques de niveau recherche, publiés ou non, émanant des établissements d'enseignement et de recherche français ou étrangers, des laboratoires publics ou privés.

\section{(c)(1)}

Distributed under a Creative Commons Attribution| 4.0 International License 


\title{
Une évaluation expérimentale des modes électroniques de résolution des litiges
}

\author{
Yannick Gabuthy * \\ Nicolas Jacquemet **
}

\begin{abstract}
Résumé. Le développement très important de l'Internet et du commerce électronique s'est accompagné de l'émergence de modes électroniques de résolution des litiges. L'objectif de l'article est d'évaluer expérimentalement l'efficacité économique d'une procédure novatrice, la négociation électronique, qui intègre la technologie comme acteur actif dans le traitement des conflits : les propositions des parties sont reçues et évaluées par un logiciel informatique qui introduit un mécanisme de convergence dans la négociation et détermine les modalités d'un accord selon une règle prédéfinie. Les résultats montrent que les parties sont incitées à exploiter stratégiquement ce mécanisme de convergence afin d'accroître leurs gains, ce qui limite largement l'efficacité de la procédure. Cependant, la menace de désaccord liée à un accroissement du degré de conflit opposant les parties semble limiter cet effet pervers, incitant ces dernières à la conciliation. Les implications de ces résultats permettent de discuter du rôle potentiel de la régulation publique et des mécanismes de réputation dans le monde virtuel.
\end{abstract}

\section{AN EXPERIMENTAL INVESTIGATION OF ONLINE DISPUTE RESOLUTION}

Abstract. The development of the Internet and electronic commerce has resulted in the emergence of Online Dispute Resolution. The aim of the paper is to evaluate experimentally the economic performance of an innovative settlement method, called automated negotiation, which inserts the technology as a third party in the conflicts: the parties' proposals are received and evaluated by an automated algorithm which provides a convergence mechanism in the bargaining process and gives a decision according to a given settlement rule. The results show that the parties are incited to exploit strategically the convergence mechanism in order to increase their payoffs, which limit the ability of the procedure to generate efficiency. However, when the threat that a disagreement occurs is more credible (due to a deeper conflict), this perverse effect is reduced since the parties are incited to adopt a more concessionary behavior. The implications of these results are then used to discuss the potential role of public regulation and reputation mechanisms in virtual world.

Classification JEL : C78, C91, D74, K41.

* Université Nancy 2 et BETA (CNRS), 13 place Carnot, 54035 Nancy, France. Yannick.Gabuthy@univ-nancy2.fr.

** Université Paris 1 Panthéon-Sorbonne et Ecole d'économie de Paris. Centre d'Economie de la Sorbonne, Bureau 420, 106 Bd. de l'hôpital, 75013 Paris, France. Nicolas.Jacquemet@univ-paris1.fr.

Nous tenons à remercier Sandra Cavaco, Nadège Marchand et Romain Zeiliger pour leur aide précieuse. Les expériences ont été réalisées grâce au soutien financier du CNRS et du Commissariat Général au Plan dans le cadre du projet « Société de l'information ». 

«The notion that most people want black-robed judges, well-dressed lawyers, and fine panelled courtrooms as the setting to resolve their dispute is not correct.
People with problems, like people with pains, want relief, and they want it as quickly and inexpensively as possible. »

Warren E. Burger, former Chief Justice, United States Supreme Court.

\section{INTRODUCTION}

La transformation par Internet des technologies de communication a entraîné une modification des conditions d'échange sur le marché. La nature ubiquitaire et universelle du réseau a notamment fait dire à nombre d'économistes qu'Internet constituait une place de marché globale et virtuelle liant directement l'offre et la demande et fonctionnant de manière quasi-walrasienne (Shapiro et Varian [1999]). Cependant, même si le succès d'Internet en tant qu'outil de communication est indéniable, il ressort de nombreuses études que le commerce électronique ne connaît pas le développement promis à ses débuts (OCDE [2003]). ${ }^{1}$ De fait, selon l'Eurobaromètre de novembre 2002, seulement $23 \%$ des internautes européens déclarent avoir déjà acheté un produit en ligne, dont seulement 5\% régulièrement et $18 \%$ occasionnellement (Dayan et Heitzmann [2006]). La raison la plus souvent invoquée de l'essor limité du commerce électronique est le risque inhérent à la transaction en ligne, risque qui s'explique largement par « l'insécurité juridique » caractérisant le réseau. En effet, la plupart des transactions sont par nature internationales, ce qui limite fortement les possibilités de recourir aux règles de droit fondées sur le principe de territorialité. La désignation d'une juridiction compétente est à cet égard le problème principal auquel est confronté le cadre juridique traditionnel en matière de commerce électronique (Deffains et Fénoglio [2001]). En outre, de nombreux différends impliquant des consommateurs en ligne sont des litiges de faibles valeurs monétaires, ce qui remet en cause l'intervention possible des tribunaux. En effet, la valeur limitée de l'enjeu économique du litige rend la durée et le coût d'une procédure juridique disproportionnés. A titre d'exemple, selon l'INSEE, la dépense annuelle moyenne d'un consommateur français sur Internet était seulement de 520 Euros en 2002. La recherche d'un système de régulation approprié du commerce électronique est ainsi passée par un élargissement du champ du juridique et une remise en cause des approches traditionnelles du droit (Reidenberg [1997]). Depuis la fin des années 90, des approches de régulation décentralisée (privée) et « déjudiciarisée » ont émergé : les modes électroniques de résolution des litiges (Online Dispute Resolution). Ces nouveaux mécanismes tirent spécifiquement parti de l'ubiquité du réseau et souhaitent procurer une protection appropriée aux protagonistes en se modelant sur l'environnement électronique : les litiges sont résolus comme les transactions sont effectuées, par Internet. De manière générale, les modes électroniques de résolution des conflits se distinguent selon le degré de contrôle dont disposent les parties dans le processus de règlement du différend et peuvent être regroupés en trois catégories (Katsh et Rifkin [2001], Rule [2002]) :

1/ L'arbitrage : une tierce partie (l'arbitre) est en mesure d'imposer une issue aux parties, issue que ces dernières devront respecter. A titre d'exemple, le centre d'arbitrage de l'OMPI (Organisation Mondiale de la Propriété Intellectuelle) est la principale institution de règlement des litiges liés à l'enregistrement et l'utilisation des noms de domaine de l'Internet (.com, .org, ...), dont la gestion est assurée par l'ICANN (Internet Corporation for Assigned Names and Numbers). ${ }^{2}$

2/ La médiation : la tierce partie (le médiateur) ne peut qu'assister les protagonistes vers une issue mutuellement avantageuse sans être en mesure de l'imposer. Depuis mars 2000, le site d'enchères eBay permet aux utilisateurs de recourir à une procédure de médiation (SquareTrade) en cas de litige relatif à l'échange d'un bien sur sa plate-forme.

3/ La négociation : cette procédure se fonde sur la recherche d'une transaction sans l'intervention d'un tiers dans le processus (les parties soumettent leurs propositions monétaires via un logiciel informatique qui leur fournit un mécanisme de convergence). Le centre de négociation Cybersettle est particulièrement sollicité dans les litiges liés au droit des assurances afin de faciliter notamment les négociations entre compagnies et assurés.

De manière générale, l'émergence de ce type de procédure semble constituer un moyen de fonder la confiance dans l'échange transfrontalier: en cas de différend, le consommateur a la possibilité de recourir à un mécanisme simple, rapide et peu coûteux dont le fonctionnement n'est pas sujet aux limites de la justice traditionnelle. En effet, la résolution du conflit est fondée sur les préférences des individus et ne requiert pas leur présence physique. La localisation légale et l'anonymat des parties ne sont donc pas des obstacles au règlement du différend. De fait, plusieurs institutions ont appelé au développement de tels mécanismes. A titre d'exemple, l'article 17 de la proposition de

\footnotetext{
1 L'OCDE définit le commerce électronique comme étant la vente ou l'achat de biens ou de services effectué par une entreprise, un particulier, une administration ou toute autre entité publique ou privée, et réalisé au moyen d'un réseau électronique.

${ }^{2}$ La majeure partie des litiges sur les noms de domaine concerne le « cybersquattage » (c'est-à-dire le dépôt abusif d'un nom déjà protégé par d'autres droits).
} 
directive européenne relative au commerce électronique invite les états membres à permettre «l'utilisation effective des mécanismes de résolution extrajudiciaires, notamment par les voies électroniques appropriées. ».33

Dans cet article, nous centrons l'analyse sur la négociation électronique, cette procédure étant la plus intéressante et la plus novatrice, de par l'absence de tiers humain dans le processus de résolution du conflit. En outre, les mécanismes d'arbitrage et de médiation ont largement été étudiés dans la littérature économique. ${ }^{4}$ Le fonctionnement de la procédure est le suivant : le processus de résolution commence lorsque le plaignant (i.e. la victime d'un préjudice) contacte un centre de négociation électronique, qui est un organisme privé, indépendant et spécialisé dans la résolution des litiges, afin de lui faire part du problème survenu lors de la transaction. Une fois la plainte déposée, le centre contacte le défendeur (i.e. l'auteur du dommage) afin de l'informer de la volonté du plaignant de résoudre le litige via la négociation électronique. Le recours à la procédure nécessitant l'accord des deux parties, le défendeur ne peut être contraint de participer à la résolution du conflit. Par simplicité et afin de centrer l'analyse sur les implications stratégiques du mécanisme de résolution proprement dit, nous supposerons cependant que la contrainte de participation du défendeur est satisfaite. ${ }^{5}$ Chacune des parties fait alors simultanément, via Internet, une proposition monétaire pour le règlement du litige, propositions analysées par un logiciel informatique qui détermine l'occurrence d'un accord et les termes de cet accord selon les modalités suivantes :

1/ Si les propositions sont compatibles (l'offre du défendeur est supérieure ou égale à la demande du plaignant), le litige est réglé et le montant versé par le défendeur correspond à la demande du plaignant.

2/ Si les propositions sont incompatibles mais suffisamment proches l'une de l'autre (la demande du plaignant est supérieure à l'offre du défendeur mais l'offre du défendeur augmentée d'un certain pourcentage est supérieure ou égale à la demande du plaignant), le litige est réglé et le montant versé par le défendeur correspond à la moyenne des deux propositions. Ce pourcentage, que nous nommons facteur de convergence, est de connaissance commune entre les parties et défini ex ante par le centre de résolution. Il est à noter que sa valeur est variable selon le centre considéré, bien qu'elle soit en général fixée à $30 \%$.

3/ Si les propositions sont trop éloignées l'une de l'autre, le litige n'est pas résolu.

Contrairement à une négociation simple, cette procédure fournit aux parties une possibilité supplémentaire de parvenir à un accord même si leurs propositions ne sont pas compatibles (via l'issue 2/), à condition naturellement que la divergence entre ces offres ne soit pas trop importante. L'existence de cette «marge de manœuvre » supplémentaire devrait ainsi accroître la probabilité de résolution du conflit, tout en laissant la liberté aux protagonistes de parvenir à un accord par eux-mêmes (via l'issue 1/). Cependant, ces avantages affichés par la profession juridique quant à l'efficacité de cette procédure reposent sur des éléments essentiellement intuitifs et manquent de fondements microéconomiques. De plus, l'absence de données empiriques, liée à l'émergence récente de ce type de mécanisme, ne permet pas de soumettre ces affirmations à réfutation. L'objectif de notre article est ainsi d'apporter des réponses quant à la pertinence de cette procédure : dans quelle mesure ce mécanisme favorise-t-il l'obtention d'un accord ? La méthode expérimentale est mobilisée afin de recréer, dans un environnement contraint et contrôlé, la procédure de négociation électronique et de produire les données nécessaires aux tests des prédictions théoriques du modèle. Le recours à l'outil expérimental se justifie pleinement ici dans la mesure où l'accessibilité à des données naturelles qui seraient fournies par les centres de résolution des conflits est particulièrement limitée, de par la confidentialité qui caractérise leur fonctionnement.

L'expérience consiste à mettre en relation un plaignant et un défendeur qui peuvent parvenir à un accord sur la compensation monétaire versée par ce dernier. Conformément à la procédure de négociation électronique, les propositions ne sont pas faites à la partie adverse mais à un logiciel qui détermine l'issue de la négociation et le montant de cette compensation selon une règle prédéfinie. Notre objectif est non seulement d'étudier le rôle du facteur de convergence mais aussi l'influence du degré initial du conflit sur les comportements individuels et l'issue du litige. Contrairement aux résultats attendus, il apparaît qu'un accroissement du facteur de convergence n'améliore pas la probabilité qu'un accord soit obtenu à l'issue de la négociation. En effet, l'existence de ce facteur incite les parties à formuler des propositions extrêmes lors de leur interaction. Les individus ont collectivement intérêt à utiliser raisonnablement la marge d'erreur fournie par le système informatique mais ont des incitations individuelles à l'exploiter stratégiquement afin d'accroître leurs gains dans l'échange, ce qui limite largement l'efficience de la

\footnotetext{
3 Directive 2000/31/CE du Parlement Européen et du Conseil du 8 juin 2000.

${ }^{4}$ Voir notamment Chappe [2001] et Wall et al. [2001].

5 Le coût monétaire du recours à la procédure est variable selon les centres de négociation électronique. Cependant, il a généralement deux composantes : des frais liés au dépôt de la plainte (supportés par le plaignant) et des frais occasionnés par la résolution du conflit (supportés par les deux parties en cas d'obtention d'un accord).
} 
procédure. Cependant, l'ampleur initiale du conflit influence la manière dont les parties appréhendent le mécanisme de résolution. La menace accrue d'un désaccord liée à une augmentation du degré de conflit opposant les parties semble limiter son effet pervers et rétablir l'efficacité de la procédure : lorsque l'ampleur du conflit s'accroît, les parties sont incitées à adopter un comportement plus conciliant lors de la négociation et à utiliser la marge d'erreur qui leur est fournie de manière plus raisonnable.

De manière générale, le logiciel informatique semble jouer le rôle d'une tierce partie dont la présence détermine le comportement stratégique des individus en conflit. Nous retrouvons ainsi certaines conclusions de la littérature économique relative à la procédure d'arbitrage. En effet, la présence d'une tierce personne «dans l'ombre des négociations » réduit les coûts liés à un échec de ces dernières (dans la mesure où un accord sera, le cas échéant, imposée par cette tierce partie) et limite ainsi les incitations des protagonistes à trouver un accord par eux-mêmes. La présence de ce «chilling effect», démontrée théoriquement (Farber [1981]), est corroborée par les travaux empiriques (Anderson [1981] ; Ashenfelter et al. [1992] ; Dickinson [2004]) : le taux d'échec des négociations est plus élevé en cas de recours possible à la procédure d'arbitrage dans la mesure où la divergence des propositions émises par les parties est plus importante. D'autres auteurs ont montré expérimentalement que cet effet entraîne une forme de dépendance à l'arbitrage («narcotic effect») : le fait d'avoir eu recours à un arbitre dans le passé incite les parties à y recourir à nouveau dans le futur (Bolton et Katok [1998]).

La section 2 est consacrée à la présentation du protocole expérimental qui décrit les paramètres utilisés pour l'expérience et les traitements effectués. Les prédictions théoriques sont également développées dans cette section. Les résultats expérimentaux et l'analyse économétrique relatifs au comportement des individus et à la résolution du litige sont analysés dans la section 3 . La dernière section donne des remarques conclusives.

\section{PRESENTATION DE L'EXPERIENCE}

\section{Cadre théorique}

Notre réflexion est menée dans le contexte d'un litige opposant la victime d'un préjudice (le plaignant, noté $P$ ) et l'auteur présumé du dommage (le défendeur, noté $D) .6$ La réalisation d'un accord consiste pour le plaignant à abandonner sa plainte moyennant un paiement couvert par le défendeur. Chaque partie $i$ est caractérisée par une valeur de réserve notée $v_{i}(i=D, P)$, qui est une information privée tirée dans une distribution uniforme de support $\left[\underline{v}_{i} ; \bar{v}_{i}\right] \cdot{ }^{7} \mathrm{La}$ valeur de réserve du plaignant est la valeur monétaire minimale qu'il est prêt à accepter pour annuler sa plainte tandis que celle du défendeur est le montant maximum qu'il est prêt à verser pour obtenir un accord. A cet égard, la valeur privée $v_{i}$ peut notamment représenter l'option extérieure du joueur $i$ : $v_{P}$ est le montant que le plaignant estime obtenir s'il recourt, par exemple, à une autre procédure de résolution des conflits, tandis que $v_{D}$ est le montant que le défendeur anticipe devoir payer dans le cadre d'une telle procédure. L'information incomplète supposée ici caractérise la plupart des litiges : le plaignant peut être mieux informé sur l'importance de son préjudice et le défendeur peut détenir plus d'éléments concernant la détermination de sa faute éventuelle. Dans ce contexte, il est à noter que les valeurs de réserve des parties sont en réalité certainement partiellement corrélées dans la mesure où elles dépendent notamment de l'origine et de l'importance du litige. L'hypothèse de valeurs privées indépendantes a été faite ici par souci de simplicité et pourrait être relâchée dans une extension de cette analyse.

Le jeu de négociation consiste alors, pour chaque partie, à proposer un montant monétaire, noté $b_{i}$, qui détermine l'issue de la négociation. Sur la base de ces propositions, l'obtention d'un accord repose sur une procédure électronique qui évalue la compatibilité des offres et détermine le prix de transaction selon la règle définie en introduction. Trois cas doivent être distingués à cet égard :

i) Les propositions sont compatibles (i.e. $b_{D} \geq b_{P}$ ). Le litige est alors résolu et le montant versé par le défendeur correspond à la demande du plaignant : $b=b_{P}$;

ii) Les propositions sont incompatibles mais suffisamment proches l'une de l'autre au regard du facteur de convergence $\delta$ (i.e. $\left.b_{D}(1+\delta) \geq b_{P}>b_{D}\right)$. Un accord est alors obtenu au prix de transaction $b=\left(b_{P}+b_{D}\right) / 2 ;$

iii) Les offres sont incompatibles et trop éloignées l'une de l'autre au regard du facteur de convergence (i.e. $\left.b_{D}(1+\delta)<b_{P}\right)$. Aucun accord n'émerge dans ce cas.

\footnotetext{
${ }^{6}$ La modélisation utilisée suit Gabuthy [2004].

7 Bien que restrictif, le recours à la loi uniforme est une hypothèse standard dans la littérature sur la négociation en information incomplète (Chatterjee et Samuelson [1983]).
} 
Le facteur de convergence $\delta \in[0,1)$ est de connaissance commune entre les parties et fixé ex ante par le centre de négociation électronique. Il représente la marge de manœuvre supplémentaire accordée aux parties pour parvenir à un accord et permet ainsi d'élargir la zone de résolution des conflits au-delà des accords qui résultent d'offres compatibles.

Les gains des parties résultent de l'accord conclu selon les règles exposées ci-dessus. En cas de résolution du conflit, le gain correspond à la différence entre le prix de transaction reçu ou versé et la valeur de réserve associée au litige. En l'absence d'accord, les gains des deux parties sont supposés nuls. ${ }^{8}$ Formellement, les fonctions de paiement s'écrivent donc :

$$
\begin{gathered}
\phi_{P}\left(b_{P}, b_{D} ; v_{P}, v_{D} ; \delta\right)=\mid \begin{array}{l}
b_{P}-v_{P} \text { si } b_{D} \geq b_{P} \\
\left(b_{P}+b_{D}\right) / 2-v_{P} \text { si } b_{D}(1+\delta) \geq b_{P}>b_{D} \\
0 \text { si } b_{D}(1+\delta)<b_{P}
\end{array} \\
\phi_{D}\left(b_{P}, b_{D} ; v_{P}, v_{D} ; \delta\right)=\mid \begin{array}{l}
v_{D}-b_{P} \text { si } b_{D} \geq b_{P} \\
v_{D}-\left(b_{P}+b_{D}\right) / 2 \text { si } b_{D}(1+\delta) \geq b_{P}>b_{D} \\
0 \text { si } b_{D}(1+\delta)<b_{P}
\end{array}
\end{gathered}
$$

Dans ce jeu, l'individu $i$ détermine sa proposition en fonction de sa valeur de réserve et de sa croyance sur la valeur de réserve de la partie adverse. Le joueur $i$ emploie alors une stratégie de meilleure réponse si, pour chaque valeur de réserve $v_{i}$, sa proposition $b_{i}$ est la meilleure réponse à la stratégie de son adversaire. La stratégie de meilleure réponse du joueur $i$ est ainsi définie par le programme de maximisation suivant :

$$
\max _{b_{i}} \Pi_{i}=E \phi_{i} \quad(i=D, P)
$$

où $E \phi_{i}$ constitue l'espérance de gain de l'individu $i$.

La résolution de ce programme de maximisation pour chaque joueur permet d'obtenir les propositions d'équilibre des parties définies par les expressions suivantes :

$$
b_{D}^{*}\left(v_{D}, \delta\right)=\frac{2(1+\delta)}{\delta^{2}+4 \delta+2} v_{D} \text { et } b_{P}^{*}\left(v_{P}, \delta\right)=\frac{2(1+\delta)}{(2+\delta)^{2}} v_{P}+\frac{4(1+\delta)^{3}}{(2+\delta)^{2}\left(\delta^{2}+4 \delta+2\right)} \bar{v}_{D}
$$

Il est à noter que ces comportements d'équilibre peuvent différer de l'efficience qui consiste, pour chaque partie, à proposer un montant correspondant à sa valeur de réserve. Ces stratégies de révélation parfaite s'écrivent formellement $: b_{i}^{e}=v_{i}(i=D, P)$.

Le facteur de convergence a deux effets opposés sur la zone d'accord qui est définie par l'inégalité suivante:

$$
b_{D}(1+\delta) \geq b_{P}
$$

- $I^{e r}$ effet : Conformément au raisonnement qui a dicté le développement de la procédure de négociation électronique, le facteur de convergence donne aux parties une chance supplémentaire de résoudre le litige et accroît donc la zone d'accord, toutes choses égales par ailleurs. Il est en effet trivial de montrer que $\delta$ a un impact positif sur le terme de gauche de l'inégalité (5).

- $2^{\text {ème }}$ effet : Cependant, dans le même temps, l'existence de ce facteur modifie le comportement d'équilibre du défendeur en l'incitant à devenir plus agressif dans la négociation (diminuant la compensation monétaire qu'il propose) :

$$
\frac{\partial b_{D}^{*}\left(v_{D}, \delta\right)}{\partial \delta}=\frac{-2\left(\delta^{2}+2 \delta+2\right)}{\left(\delta^{2}+4 \delta+2\right)^{2}} v_{D}<0
$$

Le plaignant peut de son côté être incité à diminuer sa demande, ce qui est favorable à la résolution du conflit.

\footnotetext{
${ }^{8}$ En effet, nous considérons, par souci de simplicité, que la procédure n'est pas coûteuse pour les parties. Voir Gabuthy (2004) pour une discussion approfondie des hypothèses effectuées et simplifications retenues.
} 
Cependant, cette pression à la baisse est valable uniquement pour des valeurs très élevées du facteur de convergence, valeurs qui n'existent pas dans les procédures réelles de négociation électronique. En effet, considérant par exemple $v_{P}$ $=40$ et les deux valeurs de $\delta$ retenues dans l'expérience (i.e. $\delta=0$ et $\delta=0.3$ ), nous obtenons $b_{P}^{*}(40,0)=70$ et $b_{P}^{*}(40,0.3)=70.09$. Pour ces valeurs de paramètre, le facteur de convergence opère donc bien une pression à la hausse sur la demande du plaignant.

Dans la détermination de leurs propositions, les individus font faces à un arbitrage : en adoptant une stratégie agressive, correspondant à une demande plus élevée du plaignant ou une offre plus faible du défendeur, le joueur considéré augmente son gain si le litige est réglé mais réduit la probabilité de parvenir à un accord. Inversement, en adoptant une stratégie plus conciliante, une partie augmente la probabilité de résolution du litige mais réduit le montant qu'elle obtient si un accord est effectivement obtenu. Le facteur de convergence, fournissant une marge d'erreur aux protagonistes, a un impact drastique sur cet arbitrage : les individus, de par cette «protection », ne sont pas incitées à adopter un comportement raisonnable qui supposerait qu'ils fassent des propositions proches de leurs valeurs de réserve. En d'autres termes, lorsque $\delta$ est positif, les individus sont moins accommodants durant la négociation car la menace de désaccord est moins crédible. La marge d'erreur introduite par ce facteur induit donc un effet pervers : lorsque $\delta$ augmente, l'accroissement marginal du profit associé à une proposition plus agressive fait plus que compenser la perte potentielle liée à la probabilité accrue de désaccord. En conclusion, l'effet global du facteur de convergence sur la probabilité de résolution du litige n'est pas significatif dans la mesure où le gain en efficience dû à l'augmentation de la zone d'accord potentielle ( $l^{\text {er }}$ effet) est compensé par la perte d'efficience liée au comportement stratégique des parties ( $2^{\text {ème }}$ effet).

Ce jeu de négociation constitue une représentation stylisée des procédures de négociation électronique utilisées par les centres de résolution de conflits en ligne. Il forme la base du test expérimental que nous proposons.

\section{Protocole expérimental}

L'expérience oppose un plaignant et un défendeur, cette désignation étant effectuée de manière aléatoire. A chaque partie est attribuée une valeur de réserve, tirée dans une distribution uniforme de support $\left[\underline{v}_{i} ; \bar{v}_{i}\right]$. Seules les bornes de la distribution sont de connaissance commune, les valeurs de réserve constituant quant à elles une information privée pour les participants. Au cours d'une période, les décisions des parties consistent à proposer simultanément un prix $b_{i}$. La conclusion d'un accord est alors soumise aux règles énoncées ci-dessus et les gains des parties sont calculés selon les fonctions (1) et (2).

L'impact respectif du facteur de convergence et de l'ampleur du litige est évalué à l'aide de quatre traitements, correspondant au croisement de deux couples de paramètres. D'une part, nous considérons deux ensembles de supports pour les valeurs privées des parties. Un premier traitement reflète une situation faiblement conflictuelle en attribuant des valeurs privées telles que :

$$
\text { - Conflit Faible : } v_{D} \in\{40,41, \ldots, 100\} \text { et } v_{P} \in\{0,1, \ldots, 60\}
$$

Un deuxième traitement reflète au contraire une situation de conflit élevé :

$$
\text { - Conflit Elevé : } v_{D} \in\{20,21, \ldots, 100\} \text { et } v_{P} \in\{0,1, \ldots, 80\} \text {. }
$$

Nous mettons d'autre part en évidence le rôle joué par le facteur de convergence en considérant d'abord une situation où la résolution des litiges repose uniquement sur la compatibilité des propositions :

$$
\text { - Négociation «Pure» }: \delta=0 ;
$$

Puis en considérant la procédure de négociation électronique qui élargi la zone d'accord pour des offres incompatibles mais relativement proches l'une de l'autre :

\section{- Négociation avec facteur de convergence : $\delta=0.3$.}

La combinaison de ces situations défini quatre traitements expérimentaux sous lesquels sont observés les comportements d'offre et de demande, et la résolution des conflits.

L'analyse empirique répond à un double objectif. Il s'agit d'une part d'évaluer la capacité de la procédure de négociation à encourager la révélation des valeurs privées des parties et, en particulier, à mettre en évidence l'impact du facteur de convergence sur les stratégies suivies, en comparant les comportements de proposition observés aux 
prédictions théoriques décrites ci-dessus. Une simple application des stratégies d'équilibre présentées dans l'équation (4) aux traitements décrits ci-dessus permet de formuler les hypothèses théoriques suivantes qui seront ainsi testées dans le cadre de l'expérience.

\section{Hypothèse 1 : Quelle que soit l'ampleur du conflit, le comportement du défendeur est :}

(a) Négociation pure : $b_{D}^{*}\left(v_{D}, 0\right)=v_{D}$;

(b) Négociation avec facteur de convergence : $b_{D}^{*}\left(v_{D}, 30\right)=0.79 v_{D}$.

Le comportement du défendeur est ainsi parfaitement révélateur en situation de négociation pure. En revanche, lorsque la résolution du conflit repose sur un facteur de convergence strictement positif, le défendeur adopte une stratégie de sous-évaluation de sa valeur privée.

Hypothèse 2 : Quelle que soit l'ampleur du conflit, le comportement du plaignant est :

(a) Négociation pure : $b_{P}^{*}\left(v_{P}, 0\right)=0.5 v_{P}+50$;

(b) Négociation avec facteur de convergence : $b_{P}^{*}\left(v_{P}, 30\right)=0.49 v_{P}+50.49$.

Le plaignant adopte systématiquement une stratégie de surévaluation de sa valeur privée. Le facteur de convergence induit une légère inflexion de cette stratégie.

Le but ultime des procédures de négociation électronique reste cependant, d'autre part, la résolution des litiges dont les valorisations par chacune des parties sont compatibles entre elles. Si les comportements individuels constituent le facteur déterminant de l'obtention d'un accord, celle-ci dépend également de la stratégie conjointe des parties aux litiges. Le second objectif est ainsi d'évaluer l'influence de l'ampleur du conflit et du facteur de convergence sur la probabilité de résolution des litiges.

\section{Hypothèse 3 : La probabilité qu'un accord soit conclu est décroissante de l'ampleur du conflit.}

Ce résultat provient de la diminution de la zone d'accord potentielle liée à la disjonction des intervalles sur lesquels sont distribuées les valeurs privées individuelles. En effet, lorsque l'ampleur du conflit est élevée, la probabilité que $v_{P}$ soit supérieure à $v_{D}$ est plus faible, alors même que le comportement d'équilibre des parties n'est pas modifié. ${ }^{9}$ Ces dernières ont ainsi moins de chances de parvenir à un accord lors de la négociation. Cependant, une situation potentiellement plus conflictuelle devrait intuitivement inciter les protagonistes à être plus conciliants dans la résolution du conflit. D'après les hypothèses précédentes, l'existence du facteur de convergence génère un comportement individuel sous-efficient dans la mesure où ce paramètre constitue une protection et rend la «menace » d'un désaccord moins crédible. Or, lorsque l'ampleur du conflit augmente, cette menace gagne en crédibilité, toutes choses égales par ailleurs. L'arbitrage auquel sont confrontées les parties pourrait ainsi basculer en faveur de la recherche d'un accord, cette recherche les incitant notamment à utiliser le facteur de convergence de manière plus efficiente afin de maximiser la probabilité de résolution du litige. Autrement dit, les parties pourraient être amenées à adopter un comportement moins agressif lorsque l'interaction est originellement plus conflictuelle.

\section{Procédures}

Les quatre traitements proposés sont réalisés dans le cadre de sessions expérimentales différentes. Au début de l'expérience, les participants se voient affecter aléatoirement - et définitivement - le statut de plaignant ou de défendeur. Après formation d'un couple, chaque période de jeu de l'expérience correspond au jeu de négociation théorique décrit plus haut et se déroule donc en cinq étapes :

Etape 1 - Les valeurs privées des parties $\left(v_{P}\right.$ et $\left.v_{D}\right)$ sont tirées aléatoirement selon une loi uniforme dont les bornes sont définies par les situations de conflit (voir section précédente);

Etape 2 - Chaque partie est informée de sa valeur de réserve ;

\footnotetext{
${ }^{9}$ En toute généralité, suivant l'équation (4), le comportement d'équilibre du plaignant dépend en partie de l'ampleur du litige, en raison de la présence de la borne supérieure de l'intervalle des valeurs privées du défendeur $\left(\bar{v}_{D}\right)$ dans sa stratégie d'équilibre. Cet aspect est cependant neutre dans le cadre des traitements proposés, où cette borne supérieure est constante entre les traitements.
} 
Etape 3 - Les parties choisissent librement et simultanément leur proposition, $b_{i}$, parmi les valeurs entières de l'ensemble $[0,100] ;^{10}$

Etape 4 - L'issue du litige est déterminée par application des règles (i) à (iii) exposées p.6 pour la valeur du facteur de convergence, $\delta$, correspondant au traitement considéré.

Etape 5 - Chaque partie est informée de l'issue du litige, du gain qui en résulte pour elle-même et, le cas échéant, des conditions de l'accord (i.e. le prix de transaction). En l'absence d'accord, le gain des deux parties est nul.

Deux sessions expérimentales, comprenant 20 participants chacune, ont été réalisées pour chaque traitement (soit un total de 8 sessions). Chaque session comporte en outre 40 périodes de décision. La composition du groupe de 2 sujets est modifiée de façon aléatoire à chaque période de jeu (protocole stranger). Ce protocole permet de respecter l'hypothèse centrale du modèle théorique selon laquelle la négociation électronique est un jeu statique et assure ainsi que les résultats d'équilibre restent valables dans les conditions de l'expérience.

Avant l'expérience, chaque participant tire au sort un numéro lui désignant l'ordinateur qui lui est attribué. Les participants ne peuvent à aucun moment avoir accès à une quelconque information concernant les autres membres de leur groupe et ne savent donc pas avec qui ils interagissent. Les instructions sont distribuées à chaque participant et lues à haute voix par l'expérimentaliste. ${ }^{11}$ Un questionnaire pré-expérimental est ensuite distribué aux sujets afin de vérifier que les règles du jeu sont correctement comprises. Le jeu ne démarre qu'une fois ce questionnaire corrigé publiquement. A la fin de l'expérience, les gains sont versés en espèces et de façon privée aux participants. La rémunération en Euros s'effectue sur la base de la somme des gains acquis au cours des 40 périodes de jeu selon un taux de conversion de 0.02 Euros pour 1 ECU (Experimental Currency Unit). Le total des gains obtenus par un participant à l'issue de l'expérience est majoré d'un montant forfaitaire de participation de 2 Euros. Le gain moyen des participants est de 14,3 Euros.

Les sessions expérimentales ont été conduites au cours du printemps 2003 au sein du laboratoire GATE (Groupe d'Analyse et de Théorie Economique) à Lyon. ${ }^{12}$ Ces sessions ont réuni un total de 160 participants (8 sessions comprenant 20 participants chacune), constitués principalement d'étudiants inscrits à l'ITECH (Institut Textile et Chimique de Lyon), à l'EM (Ecole de Management de Lyon) et à l'Ecole Centrale de Lyon. Les expériences réalisées ont ainsi permis de collecter 6400 observations.

\section{RESULTATS}

Les prédictions théoriques résumées plus haut décrivent à la fois les comportements individuels des parties dans la négociation et leur résultante en termes de résolution des conflits. Afin de tester l'ensemble de ces prédictions, l'analyse empirique suit une démarche identique en explorant les déterminants des comportements d'offre (de la part du défendeur) et de demande (de la part du plaignant) des parties dans l'expérience, puis l'influence des traitements expérimentaux sur la conclusion d'accords.

\footnotetext{
10 L'absence de contrainte sur la valeur proposée permet de ne pas exclure a priori les comportements fréquemment observés de sur- ou sous-enchère (voir, par exemple, Güth et al. [2005]).

11 Pour éviter d'éventuels effets de contexte social, les instructions ne font pas référence à une situation de résolution de conflit. Le jeu est ainsi présenté aux participants comme opposant un acheteur (le défendeur) et un vendeur (le plaignant) qui doivent parvenir à un accord sur le prix de vente (la compensation monétaire) d'un bien indivisible (la plainte). Les instructions sont disponibles sur simple demande auprès des auteurs.

${ }^{12}$ Le script de l'expérience a été réalisé sous le logiciel REGATE (Zeiliger [2000]).
} 
Graphique 1. Offres du défendeur

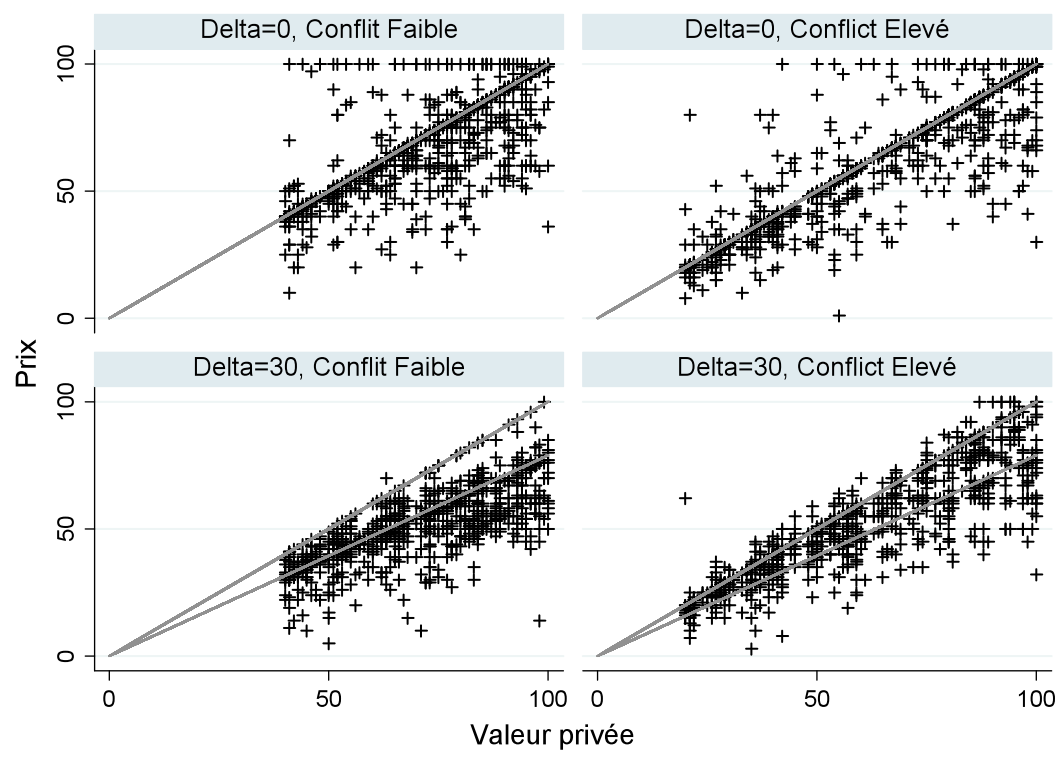

Note. Chaque point du nuage correspond à la moyenne des prix proposés par les défendeurs lorsque la valeur privée qui leur est attribuée est celle qui est reportée en ligne. Le graphique est construit séparément pour chacun des quatre traitements de l'expérience. Les droites représentent les prédictions théoriques associées à chaque traitement. La première bissectrice correspond à la stratégie efficiente $\left(b_{D}=v_{D}\right)$, la droite qui la domine correspond à la stratégie d'équilibre $\left(b_{D}=b_{D}^{*}\right)$.

\section{Comportement des parties}

A titre de statistiques descriptives, les Graphiques 1 et 2 offrent un premier aperçu des comportements respectifs des participants ayant joué le rôle de défendeur et de plaignant. Pour chacun des quatre traitements, le nuage de points est formé du niveau moyen de prix choisi (resp. offert/demandé) par les parties. Les points sont accompagnés des droites représentant les stratégies efficientes (première bissectrice) et d'équilibre déduites de la discussion théorique présentée plus haut. ${ }^{13}$ L'éventail des valeurs privées observées est déterminé par l'ampleur du conflit.

La dispersion des prix choisis par le plaignant (Graphique 2) est encadrée par les deux stratégies identifiées dans l'analyse théorique. Les stratégies empiriques apparaissent ainsi comme un compromis entre les stratégies d'équilibre et la stratégie efficiente. En comparaison du comportement observé des plaignants, les nuages des prix choisis par les défendeurs (Graphique 1) sont nettement décalés vers le bas, suggérant un éloignement vis-à-vis de la stratégie efficiente. Lorsque la négociation s'appuie sur un facteur de convergence (Graphique 1, moitié inférieure), cette tendance traduit un rapprochement vers la stratégie d'équilibre. Cette observation est particulièrement marquée lorsque le conflit qui oppose les parties est élevé (Graphique 1, quart sud-ouest).

\footnotetext{
13 En situation de négociation pure, la stratégie d'équilibre pour le défendeur concorde avec la stratégie efficiente. En conséquence, les droites coïncident dans ce cas.
} 
Graphique 2. Demandes du plaignant

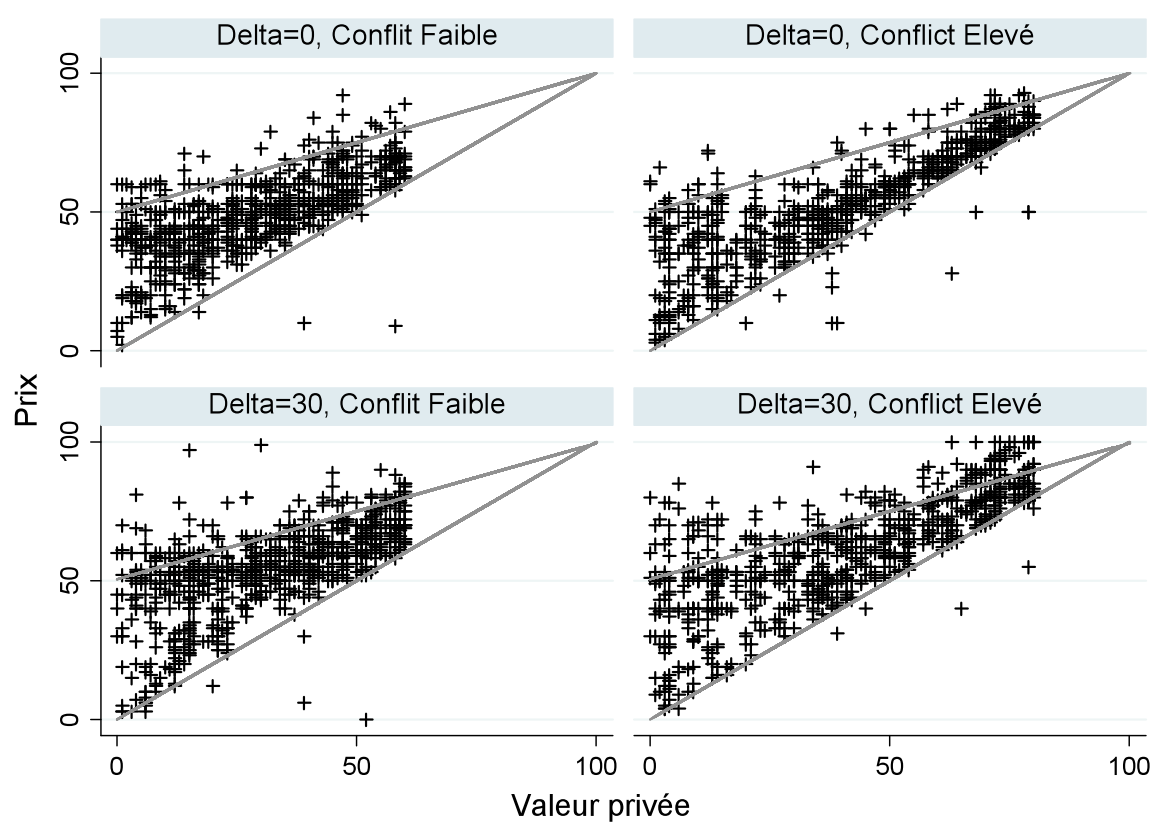

Note. Chaque point du nuage correspond à la moyenne des prix réclamés par les plaignants lorsque la valeur privée qui leur est attribuée est celle qui est reportée en ligne. Le graphique est construit séparément pour chacun des quatre traitements de l'expérience. Les droites représentent les prédictions théoriques associées à chaque traitement. La première bissectrice correspond à la stratégie efficiente $\left(b_{P}=v_{P}\right)$, la droite qui la domine correspond à la stratégie d'équilibre $\left(b_{P}=b_{P}^{*}\right)$.

Les tests formels de ces premiers éléments descriptifs reposent sur une estimation spécifique à chaque traitement des stratégies adoptées respectivement par les défendeurs et par les plaignants. L'un des avantages importants de la méthode expérimentale est en effet de fournir des observations sans erreur des valeurs privées des parties à la négociation. On peut par conséquent estimer directement, à partir des comportements observés, les coefficients des stratégies empiriques adoptées en fonction des valeurs privées. Les régressions réalisées sont ainsi de la forme :

$$
b_{i}=\beta_{\partial, C}^{i} v_{i}+\alpha_{\partial, C}^{i}+\varepsilon
$$

où $\delta=\{0 ; 0,3\}$ et $C=\{0 ; 1\}$ dénotent les traitements expérimentaux et $i=\{D, P\} .14$ Nous proposons ainsi une estimation structurelle des paramètres des stratégies adoptées empiriquement, dont les coefficients peuvent être comparés aux prédictions théoriques en termes d'équilibre comme d'efficience.

La spécification économétrique retenue est guidée par la résolution de plusieurs problèmes empiriques. D'abord, le prix proposé est, en raison du protocole de l'expérience, borné supérieurement à 100 et inférieurement à 0 . Cette variable est donc censurée, et traitée comme telle en estimant un modèle Tobit à deux bornes. ${ }^{15}$ Ensuite, la succession de 40 observations de choix pour chaque participant de l'expérience permet de contrôler les déterminants de la stratégie inhérents à des caractéristiques individuelles indépendantes de l'environnement stratégique. L'hétérogénéité inobservable est ainsi prise en compte par l'addition au modèle d'effets aléatoires individuels - de loi normale autorisant des corrélations avec le terme aléatoire. ${ }^{16}$ Nous utilisons donc un modèle à erreurs composées, le terme d'erreur $\varepsilon$ étant considéré comme résultant de deux processus aléatoires, le premier propre à chaque individu ; le second propre à chaque observation. Enfin, en raison de cette même répétition du jeu, il est vraisemblable que les comportements soient sensibles au passage du temps reflétant notamment un effet d'apprentissage lié à une plus grande familiarité avec le jeu après un certain nombre de répétitions. Cette dimension est intégrée à l'estimation en ajoutant

\footnotetext{
14 Dans cette section, on note $C=O$ le traitement avec conflit faible et $C=1$ celui avec conflit élevé.

${ }^{15}$ La stratégie suivie par les parties est ainsi considérée comme une variable continue et définie sur l'ensemble du support, mais inobservable. Le pendant empirique de cette variable latente est le prix choisi, qui n'est observable que lorsque la stratégie suivie se situe dans l'intervalle [0,100]. Le modèle Tobit repose en outre sur l'hypothèse de normalité du terme d'erreur $\varepsilon$. Voir, par exemple, Wooldridge [2002, ch.16] pour une présentation formelle du modèle et de ses propriétés.

16 L'une des limites du protocole stranger est qu'il introduit des corrélations entre les participants d'une même session. Les résultats présentés sont globalement robustes à la différenciation des stratégies en fonction de cette variable.
} 
aux variables structurelles un ensemble de variables indicatrices propres à chaque période du jeu. ${ }^{17}$

L'estimation des coefficients des stratégies adoptées empiriquement par chacune des parties permet une confrontation directe aux prédictions théoriques. Outre l'estimation, nous réalisons ainsi deux tests de spécification pour chaque traitement. Un premier test consiste à comparer les coefficients estimés à la stratégie efficiente. Il s'agit formellement du Test de Wald de l'hypothèse nulle :

$$
H_{0}^{e}:\left\{\beta_{\delta, C}^{i}=1 ; \alpha_{\delta, C}^{i}=0\right\} \forall i, \delta, C
$$

Le second test évalue la proximité des stratégies empiriques aux stratégies d'équilibre. Pour chaque traitement et chaque partie, cette évaluation est fournie par la statistique de Wald du test de l'hypothèse nulle :

$$
H_{0}^{*}:\left\{\beta_{\delta, C}^{i}=\beta_{\delta, C}^{i *} ; \alpha_{\delta, C}^{i}=\alpha_{\delta, C}^{i^{*}}\right\} \forall i, \delta, C
$$

où $\beta_{\delta, C}^{i *}$ et $\alpha_{\delta, C}^{i^{*}}$ correspondent respectivement au coefficient directeur et à l'ordonnée à l'origine de la stratégie d'équilibre du négociateur $i$ dans le traitement $(\delta, C)$ considéré. L'analyse théorique a permis d'établir que la situation de conflit $(C)$ est neutre sur la stratégie d'équilibre. Le test est donc réalisé non seulement pour chaque traitement mais également toutes situations de conflit confondues pour une procédure de résolution du litige (négociation pure ou facteur de convergence) donnée.

Appliqués successivement aux stratégies du défendeur et du plaignant, ces tests fournissent une évaluation directe de la validité des Hypothèses 1 et 2 déduites de l'analyse théorique. Les Tableaux 1 et 2 présentent les résultats de régression des stratégies estimées du défendeur et du plaignant ; accompagnés des statistiques et probabilités critiques des tests de Wald d'égalité des coefficients estimés à la stratégie efficiente et à la stratégie d'équilibre.

Pour chacune des parties, l'hypothèse nulle de conformité à l'efficience comme à l'équilibre est fortement rejetée (selon un seuil inférieur à 1\%o). Le comportement du défendeur en situation de conflit élevé lorsque la résolution du conflit s'appuie sur un facteur de convergence fait cependant exception à cette règle, confirmant la tendance suggérée par l'inspection descriptive des comportements individuels. Dans ce traitement, le comportement du défendeur ne peut pas en effet être distingué de la stratégie d'équilibre. Ce résultat très particulier mis à part (et examiné plus en détail cidessous), les Hypothèses 1 et 2 sont donc d'une façon générale formellement rejetées par les comportements expérimentaux.

\begin{tabular}{|c|c|c|c|c|c|c|}
\hline \multirow[b]{2}{*}{ Regressions } & \multicolumn{3}{|c|}{ Négociation Pure } & \multicolumn{3}{|c|}{ Facteur de Convergence } \\
\hline & Conf . Faible & Conf. Élevé & Total & Conf . Faible & Conf Élevé & Total \\
\hline & (1) & (2) & (3) & (4) & (5) & (6) \\
\hline Valeur privée & $0.82^{* * *}$ & $0.89 * * *$ & $0.87 * * *$ & $0.63^{* * *}$ & $0.79 * * *$ & $0.73^{* * *}$ \\
\hline Constante & -4.18 & $-8.90 * * *$ & $-6.77 * * *$ & -3.42 & -3.34 & $-5.21 * * *$ \\
\hline$\overline{\sigma_{\mathrm{e}}}$ & 8.41 & "6.91 & 8.31 & "5.96 & 7.27 & "6.74 \\
\hline$\sigma_{u}$ & 9.53 & 9.85 & 9.82 & 6.57 & 7.5 & 7.39 \\
\hline$\rho$ & 0.44 & 0.33 & 0.42 & 0.45 & 0.48 & 0.45 \\
\hline Log-vraisemblance & -2819.46 & -2903.03 & -5743.25 & -2675.03 & -2760.17 & -5523.61 \\
\hline \multicolumn{7}{|l|}{ Tests } \\
\hline Effi cience: stat. de Wald & 131.8 & 78.8 & 200.4 & 959.4 & 360.5 & 1144.4 \\
\hline$p$-value & 0.000 & 0.000 & 0.000 & 0.000 & 0.000 & 0.000 \\
\hline Equilibre: stat. de Wald & 131.8 & 78.8 & 200.4 & 202.7 & 3.5 & 93.9 \\
\hline$p$-value & 0.000 & 0.000 & 0.000 & 0.000 & 0.177 & 0.000 \\
\hline
\end{tabular}

Tableau 1. Analyse économétrique du comportement du défendeur

Légende. Seuils de significativité : ${ }^{*} 10 \%,{ }^{* *} 5 \%,{ }^{* * *} 1 \%$.

Note. 1600 observations sur 40 participants jouant le rôle de défendeur sont utilisées.

Moitié supérieure : Tobit à effets individuels aléatoires. La variable endogène est le prix proposé par le défendeur en : situation de négociation pure (colonne de gauche) et en présence d'un facteur de convergence (colonne de droite), selon que le conflit est faible (première colonne de chaque moitié), élevé (deuxième colonne) et indépendamment du niveau de conflit (dernière colonne). Outre la valeur de réserve, chaque régression inclut également une variable indicatrice pour chaque période de répétition du jeu, dont les coefficients ne sont pas reportés.

Moitié inférieure: Statistiques calculées et probabilités critiques des Tests de Wald d'égalité des coefficients à ceux de la stratégie efficiente (première ligne) et à ceux de la stratégie d'équilibre (seconde ligne).

\footnotetext{
${ }^{17} 39$ variables sont ainsi ajoutées à chaque modèle estimé, dont les coefficients ne sont pas reportés dans les tableaux de résultats.
} 
Cette conclusion, tirée de tests quantitatifs d'égalité des coefficients, doit cependant être nuancée par l'examen qualitatif des coefficients estimés. Pour les participants jouant le rôle de défendeurs, d'une part, la stratégie adoptée (Tableau 1) apparaît tous traitements confondus conforme à la prédiction théorique selon laquelle la droite résumant la stratégie passe par l'origine (la constante étant le plus souvent statistiquement égale à 0 et, lorsqu'elle ne l'est pas, quantitativement très faible) et est orientée selon un coefficient directeur compris, selon les traitements, entre 0.70 et 1 .

Ce coefficient tend en outre à diminuer lorsqu'un facteur de convergence soutient la résolution du litige, passant d'un ordre de grandeur de 0.85 (entre 0.82 et 0.89 suivant la situation de conflit) en situation de négociation pure à un niveau compris entre 0.63 et 0.79 en présence d'un facteur de convergence. Au regard de ces différences de comportement en réaction aux changements dans la procédure de résolution des litiges, enfin, les variations des coefficients estimés induites par différents niveaux d'intensité du conflit apparaissent de second ordre.

Les participants jouant le rôle de plaignant, d'autre part, semblent adopter une stratégie plus « agressive » que celles des défendeurs, résumée par une droite dont l'ordonnée à l'origine est fortement positive (comprise entre 18 et 35 et fortement significative) et dont la pente est de l'ordre de 0.5. L'introduction d'un facteur de convergence dans la négociation influence en outre la stratégie adoptée dans le sens prédit par la théorie. La droite ajustant la stratégie empirique tend en effet à se rapprocher de l'horizontale, sous le double effet d'un accroissement de l'ordonnée à l'origine (de l'ordre de 20 en situation de négociation pure et de 30 en présence d'un facteur de convergence) et d'une diminution de la pente (de 0.65 à 0.60 environ). Comme pour les défendeurs, enfin, l'hétérogénéité de la stratégie estimée entre situations de conflit - à procédure de résolution donnée - est de faible ampleur au regard des changements induits par la procédure elle-même.

Tableau 2. Analyse économétrique du comportement du plaignant

\begin{tabular}{|c|c|c|c|c|c|c|}
\hline \multirow[b]{2}{*}{ Regressions } & \multicolumn{3}{|c|}{ Négociation Pure } & \multicolumn{3}{|c|}{ Facteur de Convergence } \\
\hline & |Conf. Faible & Conf Éevé & Total & Conf. Faible & Conf Éevé & Total \\
\hline & (1) & (2) & (3) & (4) & (5) & (6) \\
\hline Valeur privée & $0.58 * * *$ & $0.69 * * *$ & $0.65 * * *$ & $0.54 * * *$ & $0.64 * * *$ & $0.61 * * *$ \\
\hline Constante & $23.20 * * *$ & $18.61 * * *$ & $21.52 * * *$ & $33.77 * * *$ & $35.22 * * *$ & $34.98 * * *$ \\
\hline$\sigma_{e}$ & 5.93 & 6.33 & $\overline{5.16}$ & 6.35 & 7.5 & 7.05 \\
\hline$\sigma_{u}$ & 8.31 & 8.23 & 8.42 & 9.99 & 9.27 & 9.85 \\
\hline$\rho$ & 0.34 & 0.37 & 0.27 & 0.29 & 0.4 & 0.34 \\
\hline Log-vraisemblance & -2856.27 & -2850.85 & -5738.52 & -3003.2 & -2913.54 & -5953.85 \\
\hline \multicolumn{7}{|l|}{ Tests } \\
\hline Effi cience: stat. de Wald & (497.1 & $\overline{536.5}$ & 1174.7 & 4885.3 & 640.9 & 1136.9 \\
\hline$p$-value & 0.000 & 0.000 & 0.000 & 0.000 & 0.000 & 0.000 \\
\hline Equilibre: stat. de Wald & 137.3 & 336.2 & 479.3 & 40.8 & 120.5 & 143.7 \\
\hline$p$-value & 0.000 & 0.000 & 0.000 & 0.000 & 0.000 & 0.000 \\
\hline
\end{tabular}

Légende. Seuils de significativité : ${ }^{*} 10 \%,{ }^{* *} 5 \%,{ }^{* * *} 1 \%$.

Note. 1600 observations sur 40 participants jouant le rôle de plaignant sont utilisées.

Moitié supérieure: Tobit à effets individuels aléatoires. La variable endogène est le prix proposé par le défendeur en : situation de négociation pure (colonne de gauche) et en présence d'un facteur de convergence (colonne de droite), selon que le conflit est faible (première colonne de chaque moitié), élevé (deuxième colonne) et indépendamment du niveau de conflit (dernière colonne). Outre la valeur de réserve, chaque régression inclut également une variable indicatrice pour chaque période de répétition du jeu, dont les coefficients ne sont pas reportés.

Moitié inférieure: Statistiques calculées et probabilités critiques des Tests de Wald d'égalité des coefficients à ceux de la stratégie efficiente (première ligne) et à ceux de la stratégie d'équilibre (seconde ligne).

Afin d'évaluer la pertinence statistique de ces résultats qualitatifs, nous proposons une seconde série de tests des Hypothèses 1 et 2, fondés sur des comparaisons deux à deux des stratégies estimées entre traitements, et correspondant formellement à des tests du ratio de vraisemblance (LR). Chacun de ces tests consiste à évaluer l'égalité des coefficients d'un modèle contraint à ceux d'un modèle non-contraint. Nous utilisons pour ce faire les régressions dont les résultats sont présentés dans les Tableaux 1 et 2.

Un premier test qualitatif des prédictions théoriques consiste à évaluer l'influence de la situation de conflit sur les stratégies adoptées. A procédure de résolution du litige donnée, l'intensité du conflit est en effet théoriquement neutre sur ces stratégies. Cette hypothèse est testée en comparant les modèles estimés pour chaque situation de conflit à celui qui contraint les coefficients à être égaux pour une même situation de conflit - i.e. modèles (1) et (2) contre modèle (3) et modèles (4) et (5) contre modèle (6). A l'inverse, la procédure de résolution du litige a une influence importante sur les stratégies théoriques. Cette seconde hypothèse est testée en comparant les modèles estimés pour chaque procédure de résolution - i.e. modèles (3) et (6) - à un modèle qui contraint les stratégies respectives du défendeur et du plaignant 
à être identiques entre les traitements de l'expérience. ${ }^{18}$

Les statistiques et probabilités critiques des tests sont présentées dans le Tableau 3. La procédure de résolution du litige, d'abord, semble influencer significativement les comportements observés. Les variations observées plus haut des stratégies adoptées empiriquement reflètent donc des variations significatives des comportements en réaction au changement dans la procédure de résolution des litiges. Le facteur de convergence produit ainsi les réactions stratégiques attendues de la part des parties, tendant à exploiter à leur profit l'accroissement potentiel de la zone d'accord.

Tableau 3. Effets de traitement sur le comportement des parties

\begin{tabular}{|r|c|c||c|}
\hline & \multicolumn{2}{|c||}{ Ampleur du confl it } & Facteur de \\
\hline & Delta =0 & Delta $=1$ & convergence \\
\hline Défendeur: Statistique LR & 41.5 & 176.8 & 955.5 \\
\hline$p$-value & 0.536 & 0.000 & 0.000 \\
\hline Plaignant: Statistique LR & 62.8 & 74.2 & 104.4 \\
\hline$p$-value & 0.026 & 0.002 & 0.000 \\
\hline \hline
\end{tabular}

Note. Statistiques et probabilité critiques des tests du ratio de vraisemblance pour le défendeur (moitié supérieure) et pour le plaignant (moitié inférieure). Le modèle contraint impose l'égalité des coefficients estimés de la stratégie suivie entre situations de conflits pour une procédure de résolution du litige donnée (deux première colonnes) et tous traitements confondus (dernière colonne). Le modèle non contraint laisse les coefficients libres de différer entre les traitements correspondants (resp. entre situations de conflit et entre procédures de résolution du litige)

La neutralité de l'ampleur du conflit sur les stratégies adoptées, ensuite, est acceptée sans ambiguïté en situation de négociation pure pour le défendeur, et faiblement rejetée pour le plaignant (rejetée à $5 \%$, mais non rejetée à $1 \%$ ). En présence d'un facteur de convergence, en revanche, cette hypothèse est rejetée pour les deux parties, bien que plus fortement pour le défendeur que pour le plaignant. Ces résultats permettent d'affiner les conclusions précédentes quant à l'exploitation stratégique du facteur de convergence. Si celle-ci transcende les situations de conflit (comme l'atteste l'inflexion des stratégies estimées entre les modèles (1) et (4) et (2) et (5) pour chacune des parties), l'intensité du conflit semble en effet atténuer l'incitation à se montrer plus agressif dans la négociation.

Bien que les Hypothèses 1 et 2 soient quantitativement rejetées par l'analyse statistique, les prédictions théoriques apparaissent ainsi comme un guide qualitatif satisfaisant des comportements de négociation observée. Le facteur de convergence fait en particulier l'objet d'une exploitation stratégique, par laquelle les parties accroissent le gain espéré tiré d'un accord en réaction à l'accroissement de la probabilité de résolution du litige. Si l'intensité du conflit est de peu d'effet sur les comportements en situation de négociation pure, en outre, elle semble à même de nuancer l'effet stratégique du facteur de convergence en raison de l'accroissement induit du risque de désaccord.

\section{Résolution des conflits}

Les comportements d'offre et de demande décrits dans la section précédente sont à la base des possibilités de résolution des conflits offertes par les différentes procédures. La conclusion effective d'accords dépend cependant également de la règle de résolution appliquée à ces décisions individuelles. L'élargissement potentiel de la zone d'accord offerte par le facteur de convergence peut en effet plus que compenser la réaction stratégique des parties.

Le Graphique 3 fournit un premier aperçu de cet aspect en reproduisant, pour chaque procédure, l'écart moyen observé entre les propositions des parties en fonction de l'écart entre les valeurs de réserve induites. Toutes les observations pour lesquelles la différence moyenne entre prix proposés est positive (i.e. situés au dessus de la droite

\footnotetext{
18 Ces régressions présentent un intérêt mineur en termes de compréhension des comportements empiriques et ne sont donc pas incluses aux Tableaux 1 et 2. Elles sont disponibles sur simple demande auprès des auteurs.
} 
horizontale) conduiraient à un accord selon une règle de négociation pure - par laquelle le litige n'est résolu que si l'offre domine la demande. L'efficience économique exigerait quant à elle que toutes les situations où les valeurs de réserve sont compatibles entre elles (c'est-à-dire les observations qui se situent à l'ouest de la droite verticale) conduisent à un accord.

L'écart observé entre les deux traitements rend compte de l'exploitation stratégique du facteur de convergence observée plus haut. Lorsque celui-ci détermine la résolution du litige, les offres et demandes des parties tendent à se montrer moins propices à conduire à un accord selon une règle de négociation pure. Quel que soit le traitement, les comportements observés tendent en outre à confirmer l'écart vis-à-vis d'une situation efficiente : les offres et demandes apparaissent en moyenne d'autant plus incompatibles que l'écart entre valeurs privées - bien que positif - est faible, comme l'indique la pente positive des courbes.

Graphique 3. Résolution du litige et compatibilité des offres

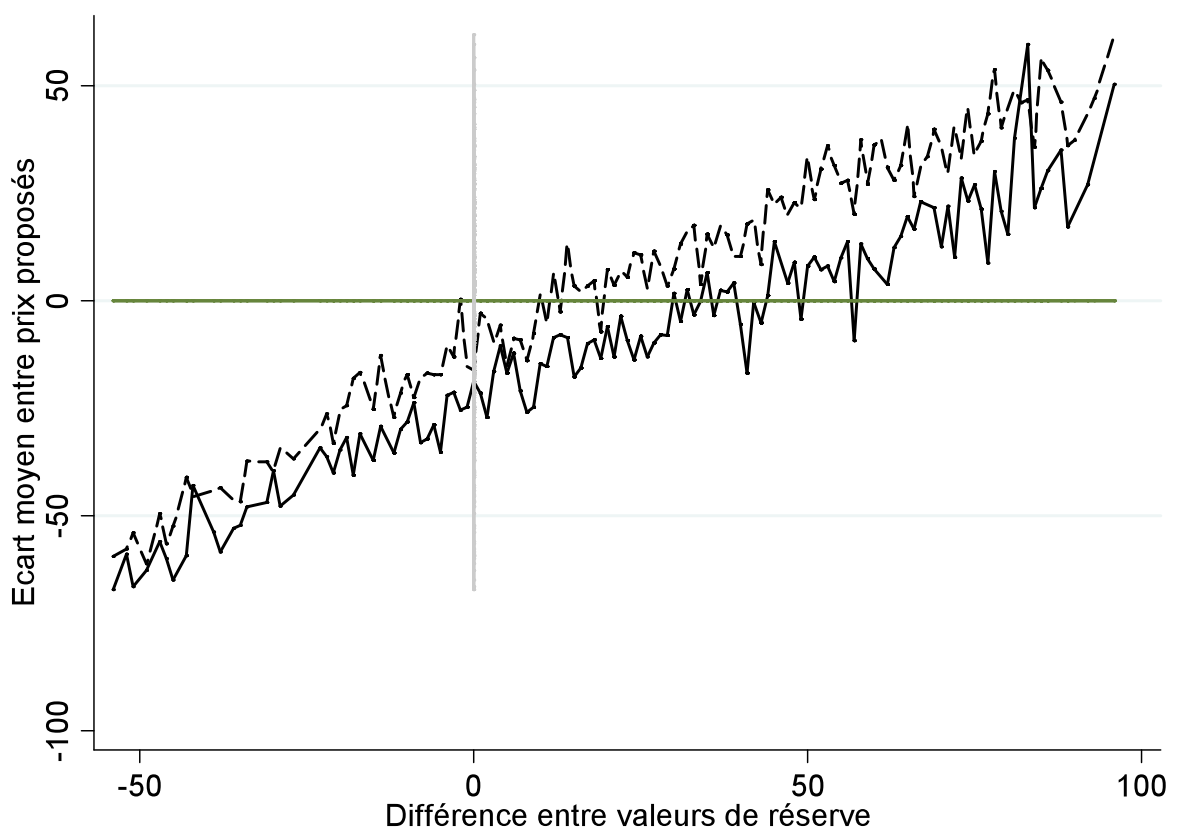

Légende. Pointillés : Traitement «négociation pure »; Trait plein : Traitement «facteur de convergence».

Note. Les droites représentent l'écart moyen entre le prix proposé par le défendeur et demandé par le plaignant (mesurée par $b_{\mathrm{D}}-\mathrm{b}_{\mathrm{P}}$ ) en fonction de la différence entre les valeurs de réserve induites $\left(\mathrm{v}_{\mathrm{D}}-\mathrm{v}_{\mathrm{P}}\right)$. La droite verticale permet de distinguer les observations dont les valeurs privées sont compatibles (à droite); la droite horizontale les observations qui conduiraient à un accord selon une règle de négociation pure (en haut).

A ce premier effet en termes d'exploitation stratégique du facteur de convergence s'ajoute celui de l'élargissement des possibilités d'accord offert par cette procédure. Le Tableau 4 présente la résultante de ces deux effets, à travers la proportion d'accords effectivement conclus (par application de la règle déterminée par le traitement expérimental).

La principale leçon à tirer de ces statistiques est que l'effet direct de l'élargissement de la zone d'accord mise en œuvre par l'introduction d'un facteur de convergence semble être de second ordre au regard du changement induit dans les stratégies adoptées par les parties. La résultante de ces deux forces tend cependant à se rééquilibrer en faveur de la première à mesure que l'intensité du conflit s'accroît. 
Tableau 4. Proportion d'accords conclus

\begin{tabular}{|c|c|c|c|c|c|c||c|c|c|}
\hline & \multicolumn{3}{|c|}{ Valeurs compatibles } & \multicolumn{3}{c||}{ Valeurs incompatibles } & \multicolumn{3}{c|}{ Ensemble } \\
\hline & C. Faible & C. Elevé & Tot. & C. Faible & C. Elevé & Tot. & C. Faible & C. Elevé & Tot. \\
\hline Négociation pure & 79.15 & 73.95 & 76.93 & 3.57 & 4.82 & 4.69 & 76.50 & 54.25 & 65.38 \\
\hline Nbre. Obs. & 772 & 572 & 1344 & 28 & 228 & 256 & 800 & 800 & 1600 \\
\hline \hline Facteur de convergence & 75.14 & 77.45 & 76.15 & 1.56 & 8.33 & 6.85 & 69.25 & 57.75 & 63.50 \\
\hline Nbre. Obs. & 736 & 572 & 1308 & 64 & 228 & 292 & 800 & 800 & 1600 \\
\hline \hline Ensemble & 77.19 & 75.70 & 76.55 & 2.17 & 6.58 & 5.84 & 72.88 & 56.00 & 64.44 \\
\hline Nbre. Obs. & 1508 & 1144 & 2652 & 92 & 456 & 548 & 1600 & 1600 & 3200 \\
\hline \hline
\end{tabular}

Note. Chaque cellule présente la proportion d'accords conclus (en \%) ainsi que le nombre d'observations sur lequel cette proportion est calculée. Les calculs sont différenciés selon la compatibilité des valeurs de réserve (compatible si $v_{D}>v_{P}$ ) et les traitements expérimentaux.

Dans l'ensemble, d'abord, l'introduction du facteur de convergence semble échouer à promouvoir la conclusion d'accords entre les parties, la proportion d'accords passant globalement de $65 \%$ à $63 \%$. Cette proportion globale mélange cependant l'effet de la résolution des conflits et celle de la disparité de compatibilité des offres inhérentes aux aléas du tirage des valeurs privées. Ce dernier effet est neutralisé en considérant les proportions d'accords conclus entre parties dont les valeurs de réserve sont compatibles entre elles (première colonne du Tableau 4). Le résultat semble robuste à ce contrôle supplémentaire, quoique de façon atténuée - la proportion d'accords entre offres compatibles passant de $77 \%$ à $76 \%$. La plus grande partie de la différence observée entre procédures de résolution du conflit provient en effet de l'influence du facteur de convergence sur la conclusion d'accords entre parties dont les valeurs de réserve sont incompatibles. Cet effet constitue une seconde source de déviation vis-à-vis de la solution efficiente, puisque l'élargissement des possibilités d'entente tend alors à contraindre la conclusion d'accords dans des situations qui n'offrent aucune possibilité de gain mutuel entre les parties.

Cet effet du facteur de convergence recouvre cependant une grande disparité entre situations de conflit. L'intensité du conflit a, par elle-même, un effet ambigu sur la résolution des litiges. A mesure que l'intensité du conflit s'accroît, la probabilité que les valeurs de réserve des parties soient incompatibles devient plus grande et la probabilité d'un accord plus petite. Cet effet se traduit globalement par une diminution importante de la proportion d'accords conclus avec l'augmentation de l'intensité du conflit (passant dans l'ensemble de $73 \%$ à 56\%), confirmant l'Hypothèse 3.

Simultanément, enfin, ce risque de désaccord tend également, comme nous l'avons vu, à discipliner les parties en encourageant une plus grande modération des prix proposés. Ce dernier effet tend à renverser l'influence du facteur de convergence sur la proportion d'accords conclus en situation de conflit élevé. Si le facteur de convergence réduit globalement la résolution des litiges, il parvient en effet à promouvoir la conclusion d'accords dans les situations où le risque de désaccord ex ante est plus élevé. ${ }^{19}$

\section{CONCLUSION}

Dans cet article, nous avons développé une analyse expérimentale afin d'étudier l'efficacité économique d'une procédure alternative de résolution des conflits, la négociation électronique. Les conclusions de Smith [1962] selon lesquelles les règles de fonctionnement du marché déterminent le comportement des agents économiques s'appliquent pleinement ici. Le mécanisme de convergence introduit dans la procédure et la prise de décision en situation d'incertitude déterminent l'environnement dans lequel les parties négocient. Cet environnement a alors un impact très important sur leurs comportements stratégiques et le règlement du différend. Les parties sont en effet incitées à exploiter la marge de manœuvre que fournit la procédure afin d'accroître leurs profits. Cependant, la menace de désaccord liée à un accroissement du degré de conflit opposant les parties semble limiter cet effet pervers, incitant ces dernières à la conciliation. Ce résultat permet de relativiser les conclusions quant à l'inefficacité de la négociation électronique dans la mesure où il met en évidence le rôle déterminant que peut avoir le facteur de convergence sur l'obtention d'un accord.

Plusieurs questions restent à aborder au regard du fonctionnement des modes électroniques de résolution des litiges et de leurs implications. Etant donné que ce fonctionnement est pleinement contractuel et que ces procédures sont

\footnotetext{
${ }^{19}$ La significativité statistique de ces conclusions a été testée par une régression de la proportion d'accords sur les variables de traitement. Les résultats de régression ne font que confirmer les statistiques descriptives présentées dans le Tableau 3 : tous les effets de traitement sont non significatifs, à l'exception de l'interaction entre situation de conflit et facteur de convergence. Ces régressions sont donc omises et disponibles sur simple demande auprès des auteurs.
} 
proposées par des entreprises privées, comment s'assurer que les parties (et notamment les défendeurs) accepteront d'y recourir ex ante et de respecter l'accord obtenu ex post. En effet, alors même que son intervention est compromise ici, seule la justice étatique (publique) a un pouvoir de contrainte quant à l'exécution d'un accord entre deux parties en conflit. A cet égard, le rôle des mécanismes de réputation existant sur les plateformes électroniques d'échange (telles que eBay ou Amazon) peut être prépondérant: la volonté de l'usager de conserver, voire d'accroître, son niveau de réputation au sein de la communauté peut l'inciter à accepter le recours à un mode électronique de résolution des litiges, à se conformer à l'accord ainsi scellé, voire à modifier son comportement de négociation lors de la résolution du conflit proprement dite. ${ }^{20}$ L'étude de l'interaction entre les modes électroniques de résolution des litiges et les mécanismes de réputation mis en place par certaines plateformes d'échange pourrait faire l'objet d'analyses expérimentales ultérieures. En ce sens, notre analyse doit être considérée comme une première étape d'investigation empirique de la résolution électronique des litiges. Suivant les arguments développés ci-dessus, des analyses théoriques et expérimentales supplémentaires devront être développées avant d'avoir une vision pertinente du fonctionnement et des implications de ces mécanismes dans leur ensemble.

\section{RÉFÉRENCES BIBLIOGRAPHIQUES}

ANDERSON John C. [1981], “The Impact of Arbitration: a Methodological Assessment”, Industrial Relations, 20 (6), p. 129-148.

ASHENFELTER Orley, CURRIE Janet, FARBER Henry et SPIEGEL Matthew [1992], “An Experimental Comparison of Dispute Rates in Alternative Arbitration Systems", Econometrica, 60 (6), p. 1407-1433.

BOLTON Gary E. et KATOK Elena [1998], "Reinterpreting Arbitration's Narcotic Effect: An Experimental Study of Learning in Repeated Bargaining", Games and Economic Behavior, 25 (1), p. 1-33.

CHAPPE Nathalie [2001], “L'analyse économique d'un mode de résolution des litiges: l'arbitrage”, Revue Française d'Economie, 15 (4), p. 187-208.

CHATTERJEE Kalyan et SAMUELSON William [1983], "Bargaining Under Incomplete Information", Operations Research, 31 (5), p. 835-851.

DAYAN Martine et HEITZMANN Raymond [2006], “Tableau de bord du commerce électronique”, Ministère délégué à l'industrie, Service des études et des statistiques industrielles.

DEFFAINS Bruno et FENOGLIO Philippe [2001], "Economie et ordre juridique virtuel”, Revue Economique, 52 (0), p. 331-347.

DICKINSON David [2004], “A Comparison of Convetional, Final-Offer, and Combined Arbitration for Dispute Resolution”, Industrial and Labor Relations Review, 57 (2), p. 288-301.

FARBER Henry S. [1981], "Splitting-the-Difference in Interest Arbitration", Industrial and Labor Relations Review, 35 (1), p. 70-77.

GABUTHY Yannick [2004], “Online Dispute Resolution and Bargaining”, European Journal of Law and Economics, 17 (3), p. 353-371.

GUTH Werner, IVANOVA-STENZEL Radosveta et WOLFSTETTER Elmar [2005], "Bidding Behavior in Asymmetric Auctions: An Experimental Study", European Economic Review, 49 (7), p. 1891-1913.

KATSH Ethan et RIFKIN Janet [2001], Online Dispute Resolution: Resolving Conflicts in Cyberspace, San Francisco: Jossey-Bass Press.

OCDE [2003], “Technologies de l'information et des communications. Perspectives de communications”, 26, p. 245-270.

REIDENBERG Joel (1997), "Governing Networks and Rule-Making in Cyberspace", in Borders in Cyberspace: Information Policy and the Global Information Structure (eds. Kahin B. et Nesson C.), Cambridge : MIT Press.

RESNICK Paul, ZECKHAUSER Richard, SWANSON John et LOCKWOOD Kate [2006], "The Value of Reputation on eBay: A Controlled Experiment", Experimental Economics, 9 (2), p. 79-101.

RULE Colin [2002], Online Dispute Resolution for Business, San Francisco: Jossey-Bass Press.

SHAPIRO Carl et VARIAN Hal R. [1999], Information Rules: A Strategic Guide to the Network Economy, Boston: Harvard Business School Press.

SMITH Vernon L. [1962], “An Experimental Study of Competitive Market Behavior”, Journal of Political Economy, 70

\footnotetext{
20 Suivant ces mécanismes, chaque participant à une transaction peut évaluer le membre avec lequel il vient de réaliser l'échange en lui attribuant une note. Toutes les notes attribuées à un membre sont additionnées et constituent ainsi son profil d'évaluation, profil qui est rendu public par affichage sur le site (Resnick et al. [2006]).
} 
(2), p. 111-137.

WALL James A. Jr, STARK John B. et STANDIFER Rhetta L. [2001], "Mediation: A Current Review and Theory Development", Journal of Conflict Resolution, 45 (3), p. 370-391.

WOOLDRIDGE, Jeffrey M. [2002], Econometric Analysis of Cross Section and Panel Data, Cambridge (MA): MIT Press.

ZEILIGER Romain [2000], "A Presentation of Regate, Internet Based Software for Experimental Economics", http://www.gate.cnrs.fr/ zeiliger/regate/regate.htm, GATE. 